\title{
Association Between Perceived Stress, Loneliness and Sleep Disorders Among Breast Cancer Patients: the Moderating Roles of Resilience
}

\section{Yue Ban}

China Medical University

\section{Mengyao Li}

China Medical College: China Medical University

He Bai

Anshan Cancer Hospital

Li Liu

China Medical College: China Medical University

\section{Zhihui Gu}

China Medical College: China Medical University

\section{Ke Zhang}

China Medical College: China Medical University

\section{Chen xin Yang}

China Medical College: China Medical University

Hui Wu ( $\nabla$ hwu@cmu.edu.cn )

Department of Social Medicine,School of Health Management, China Medical University https://orcid.org/0000-0002-4157-8023

\section{Research Article}

Keywords: breast cancer, perceived stress, loneliness, sleep disorders, resilience, moderating effect

Posted Date: February 28th, 2022

DOI: https://doi.org/10.21203/rs.3.rs-1262325/v1

License: (c) (i) This work is licensed under a Creative Commons Attribution 4.0 International License. Read Full License 


\section{Abstract}

Purpose: Sleep disorders are prevalent and often neglected among breast cancer patients. This study aimed to identify the association among perceived stress, loneliness, resilience and sleep disorders, and to further explore whether resilience could be a moderator in the association between perceived stress, loneliness and sleep disorders among Chinese female breast cancer patients.

Methods: A cross-sectional study was conducted at a tertiary hospital in Anshan, China from October 2019 to October 2020. 492 valid questionnaires were collected. Patients were asked in response to the questionnaires including the Pittsburgh Sleep Quality Index, Perceived Stress Scale-10, the Three-Item Loneliness Scale and Resilience Scale-14. The association among perceived stress, loneliness, resilience and perceived stress/loneliness $\times$ resilience interaction with sleep disorders were examined by hierarchical multiple regression analysis. The interaction was visualized by using simple slope analysis.

Results: In our study, $36.58 \%$ of the female breast cancer patients reported sleep disorders. Perceived stress and loneliness were related to sleep disorders. Resilience could moderate the relationship between them and sleep disorders. When resilience was higher, perceived stress and loneliness had a weaker impact on sleep disorders.

Conclusions: There was a high prevalence of sleep disorders and this study implied an adverse effect of perceived stress, loneliness on the sleep quality. However, resilience could moderate the association between perceived stress, loneliness and sleep disorders. More positive psychological interventions should be integrated into the prevention of sleep disorders among cancer patients.

\section{Introduction}

Breast cancer (BC) is currently one of the most common malignant tumors in women worldwide, whose incidence has been sharply increasing in recent decade [12]. After a series of diagnoses and treatments, $\mathrm{BC}$ patients suffer from pain, fatigue, anxiety and sleep disorders, giving rise to increasing the risk of allcause mortality among women [3]. Among negative health consequences, sleep disorders receive inadequate academic attention. Sleep disorders generally comprise various potential symptoms and disturbances including insomnia, nighttime sleep disruptions and excessive daytime sleepiness, etc. Some studies have shown that the incidence of sleep disorders among breast cancer survivors (BCSs) is significantly higher than that of the general population [35]. Thus, in order to relieve sleep disorders among female $\mathrm{BC}$ patients, it is vital to explore the potential influencing factors.

Psychological factors such as perceived stress can affect the sleep quality of BC patients [10]. Perceived stress refers to the cognitive, psychological and physiological reactions of individuals confronted the various life events beyond their mental endurance [21]. Previous study has shown that stress can lead to rapid progression of cancer disease and severe physical and psychological consequences for patients [3]. According to the stress-coping model, whether the stressor produces stress after acting on the individual is related to their cognitive [11]. BC patients not only have to face the threat of cancer to life, but also 
have to accept the reality of self-image disturbances in the process of treatment, which will inevitably lead to long-term distress, and are more prone to suffer from psychological stress than other cancer patients [10]. In addition, Scholars emphasized that those who have stronger stress perceptions are more prone to suffer from sleep disorders [17]. Therefore, the rising levels of perceived stress will contribute to poor sleep quality.

Loneliness is defined as subjective distress due to a discordance between expected and actual perceived social relationships, which is a relevant psychological construct correlated to the social contexts of cancer [16]. Loneliness is detrimental to cancer patients' psychological well-being and even lead to negative health outcomes including sleep disorders, anxiety and depressive symptoms [20]. Specifically, after experiencing a cancer diagnosis, BC patients are more vulnerable to have higher expectations of emotional support, whereas these expectations cannot be fully met due to subjective or objective reasons, which in turn leads to higher loneliness [2]. Matthews et al. reported that the relationship between loneliness and poor sleep quality in adults was especially performed for those who had experienced traumatic events [25]. Besides, study in individuals with cancer showed that they generally prone to moderate levels of loneliness and that would result in poorer mental health and sleep quality [7]. Hence, loneliness might be served as a risk factor of sleep disorders. However, there are few studies have directly explored the relationship between the loneliness and sleep disorders among Chinese female BC patients. Since these negative psychological factors (perceived stress and loneliness) might affect the patients' sleep quality, it is necessary to explore some positive psychological resources to cope with the adverse effect.

Resilience, as a personality trait defined by flexibility in response to changing environmental demands and the ability to recover from negative life experiences (adversity), is a psychological concept [30]. Patients with high resilience have a clearly cognition to their diseases, and more strictly stick with the treatment to avoid risks and reduce negative factors of coping style [18]. Zou et al. revealed that high resilience increased patients' positive affect and decreased negative emotions as well as relieved cancerrelated fatigue (e.g. pain, sleep disturbance) [39]. Researches have also shown that resilience acted as a moderated mediation role in the correlation between stress and negative affect in cancer survivors [26].

Previous studies have confirmed that the associations of perceived stress, loneliness with sleep quality and the correlations of resilience with the symptoms among cancer survivors. Perceived stress and loneliness might be predictors of sleep disorders [20,37]. Carli et al. have shown that resilience was regarded as a protective factor for sleep quality [5]. Nevertheless, the role of resilience as a moderating role in the relationships between perceived stress, loneliness and sleep disorders among Chinese female $\mathrm{BC}$ patients are still not be examined to our limited knowledge. Academically, resilience is not only a personality trait, but also a positive coping style and an interactive dynamic construct [30], which can adjust negative factors together with facilitating positive psychological changes and health outcomes in the process of the cancer experience [33]. 
In view of the above concerns, this study aimed to examine (1) the relationships perceived stress, loneliness with sleep disorders, and (2) to further explore whether resilience moderated the effect of perceived stress, loneliness on sleep disorders in Chinese BC patients.

\section{Methods}

\section{Participants and design}

The study was conducted at a tertiary hospital in Anshan, China from October 2019 to October 2020. All participants were from the Department of Breast Surgery. All the patients had completed surgical treatment and continued radiotherapy or chemotherapy. The inclusion criteria in our study were as follows: 1) 18 years old or above, female; 2) diagnosed with breast cancer (IIV); 3) fluent communications in Chinese and had the ability to accurately answer questions. Exclusion criteria were as follows: 1) patients had a history of psychosis in the light of medical records or were unable to read the protocol and questionnaire; 2) unwilling to be enrolled into the program.

\section{Data collection}

The data collection was completed by researchers and medical staff, of whom had underwent rigorous training before the survey. When the eligible patients were ready to be discharged from breast surgery ward, their attending physician would invited them to participate in the study. After patients signed an informed consent form, they were asked to fill out a questionnaire in a quiet room in the inpatient department. The researchers would guide participants how to fill in the questionnaire and explained item if participants had difficulty in reading or understanding. The process of collecting questionnaires had strict quality control measures to avoid possible bias. Of the 522 eligible patients, 492 patients agreed to participate and fill in completing questionnaires, with effective response rate of $94.25 \%$. The main reasons for non-participation included physical problems, mobility-impaired and not interested in this research.

\section{Measures}

\section{Measurement of sleep disorders}

The Study used the Pittsburgh Sleep Quality Index (PSQI) scale [4] to evaluate overall sleep problems of the patients; it includes 19 self-rated items scale that consists of seven dimensions: sleep duration, sleep latency, sleep disturbance, habitual sleep efficiency, sleeping medication use, daytime dysfunction, and overall sleep quality. Each component is rated on a scale of $0-3$, a global PSQI score was determined by summing the seven components scores, which ranged from 0 to 21 . The higher scores indicate lower sleep quality, and a PSQI score greater than 5 identifies an individual with sleep disorders [4]. The Chinese version had been demonstrated adequate reliability and validity in previous research [15] and Cronbach's a was 0.909 in this study.

\section{Measurement of perceived stress}


The Perceived Stress Scale-10 (PSS-10) was used to assess participants' perception of stress [6]. Each item was answered by a 5 -point Likert scale ( $0=$ "never" to $4=$ "very often"). The total score ranges from 0 to 40 with a higher score indicating higher level of perceived stress. The Chinese version of the scale had been verified to a good reliability and validity [36]. In this study, the Cronbach's alpha coefficient for the total scale was 0.702 .

\section{Measurement of loneliness}

Loneliness was measured by the 3-item version of the UCLA Loneliness Scale [19]. Each item was rated on a 3-point scale ranging from 1 (hardly ever) to 3 (often), with higher scores indicating greater loneliness. This scale had good reliability and validity among different groups [13]. The Cronbach's alpha coefficient for the total scale was 0.919 in our study.

\section{Measurement of resilience}

The resilience was measured using the US English Version of the 14-item Resilience Scale (RS-14) [34]. Items used a 7-point Likert scale ranging from "strongly disagree" to "strongly agree". The total score of the scale ranged from 14-98, with higher scores indicating higher levels of resilience. The reliability and validity of the Chinese version had been confirmed [32]. In our research, the Cronbach's alpha coefficient for the total scale was 0.969 .

\section{Demographic and clinical characteristics}

Demographic variables included age, residence, marital status, educational level, monthly income, principal caregiver and physical activity were obtained in this study. Age was divided as two groups including " $\leq 55$ years old" and " $>55$ years old". Residence was categorized as "city" and "rural". Marital status included "married/cohabitation" and "single/divorced/widow/separated". Educational level was divided as "primary/middle school", "high school" and "junior college and over". Monthly income was categorized as " $\leq 3000$ ", "3000-5000" and ">5000". Principal caregiver was divided into "spouse", "relatives" and "adult children". Physical activity was divided into "yes" and "no" two groups. Clinical factors comprised cancer stages, recurrence, chemotherapy, radiotherapy. Cancer stages were divided as "I", "II" and "III+IV" three groups. Recurrence, chemotherapy and radiotherapy were categorized as "yes" and "no" two groups, respectively.

\section{Statistical analysis}

Firstly, the demographic and clinical variables were described with number (n), percentage (\%) and mean, standard deviation (SD) as appropriate. Group comparisons of continuous variables were made by using t-test or one-way ANOVA. Secondly, the correlation between continuous variables was described by using Pearson correlation analysis. Thirdly, hierarchical multiple regression was conducted for testing the association among perceived stress, loneliness, resilience and sleep disorders together with exploring the moderating role of resilience on the association among perceived stress, loneliness and sleep disorders, respectively. In step 1 , age and principal caregiver in univariate analysis $(P<0.05)$ were added. Perceived stress, loneliness and resilience were added in step 2, respectively. Finally, the product of perceived stress, 
loneliness and resilience respectively was added in step 3 . All research variables were centralized before regression analysis to explain differences in scale scores. If the interaction effect was statistically significant, simple slope analysis was conducted to visualize the interaction term. In the present study, the Variance Inflation Factor (VIF) values $<10$, which indicated that multicollinearity was not an issue in the estimate. All the statistical analyses were performed using SPSS 21.0 (IBM, Asia Analytics Shanghai), with a two-tailed $P<0.05$ viewed as statistically significant.

\section{Results}

\section{Descriptive statistics}

Demographic and clinical characteristics of participants and group differences on sleep disorders were displayed in Table 1. In our research, of the 492 respondents, the average age of patients was $55.35 \pm 10.57$ (Mean \pm SD) years. A total of 251 (51\%) patients were cared by relatives, $184(37 \%)$ patients were cared by spouse and $57(12 \%)$ patients were cared by adult children. Among the seven demographic variables, only principal caregiver was found to be significantly correlated with sleep disorders, patients who were cared by spouse reported higher sleep disorders than those were cared by relatives and adult children $(P<0.05)$. However, there were no significant differences on age, residence, marital status, education level, monthly income, physical activity, cancer stages, recurrence, chemotherapy and radiotherapy on sleep disorders in the descriptive statistics $(P>0.05)$.

Table 1 Demographic characteristics and clinical characteristics of the study subjects $(N=492)$ and univariate analysis for the factors related to the sleep disorders 


\begin{tabular}{|c|c|c|c|c|}
\hline \multirow[t]{2}{*}{ Variables } & \multirow[t]{2}{*}{$\mathrm{N}(\%)$} & \multicolumn{3}{|c|}{ Sleep disorders } \\
\hline & & Mean $\pm S D$ & $F / t$ & $p$-value \\
\hline Age (years) & & & 0.841 & 0.401 \\
\hline$\leq 55$ & $233(47)$ & $5.44 \pm 3.36$ & & \\
\hline$>55$ & $259(53)$ & $5.19 \pm 3.29$ & & \\
\hline Residence & & & 0.403 & 0.687 \\
\hline city & $375(76)$ & $5.34 \pm 3.26$ & & \\
\hline rural & $117(24)$ & $5.20 \pm 3.54$ & & \\
\hline Marital status & & & -0.397 & 0.692 \\
\hline married/cohabitation & $495(92)$ & $5.29 \pm 3.32$ & & \\
\hline single/divorced/Separated/widow & $37(8)$ & $5.29 \pm 3.32$ & & \\
\hline Education level & & & 0.940 & 0.391 \\
\hline primary/middle school & $165(34)$ & $5.08 \pm 3.41$ & & \\
\hline high school & $222(45)$ & $5.31 \pm 3.25$ & & \\
\hline junior College and over & $105(21)$ & $5.65 \pm 3.36$ & & \\
\hline Income (Yuan per Month) & & & 1.483 & 0.476 \\
\hline$\leq 3000$ & $120(24)$ & $5.71 \pm 3.89$ & & \\
\hline $3000-5000$ & $309(63)$ & $5.16 \pm 3.18$ & & \\
\hline$>5000$ & $63(13)$ & $5.27 \pm 2.79$ & & \\
\hline Principal caregiver & & & 3.041 & 0.049 \\
\hline spouse & $184(37)$ & $5.76 \pm 3.51$ & & \\
\hline relatives & $251(51)$ & $4.96 \pm 3.14$ & & \\
\hline adult children & $57(12)$ & $5.35 \pm 3.36$ & & \\
\hline \multicolumn{5}{|l|}{ Physical activity } \\
\hline yes & $447(91)$ & $5.23 \pm 3.29$ & 1.663 & 0.097 \\
\hline no & $45(9)$ & $6.09 \pm 3.62$ & & \\
\hline Cancer stages & & & 0.463 & 0.630 \\
\hline I & $143(29)$ & $5.35 \pm 3.51$ & & \\
\hline II & $199(40)$ & $5.14 \pm 3.20$ & & \\
\hline
\end{tabular}




\begin{tabular}{|ccccc|}
\hline III+IV & $150(31)$ & $5.48 \pm 3.32$ & & \\
Recurrence & & & & \\
yes & $65(13)$ & $6.02 \pm 3.30$ & 1.855 & 0.064 \\
no & $427(87)$ & $5.20 \pm 3.32$ & & \\
Chemotherapy & & & -0.813 & 0.417 \\
\hline yes & $281(57)$ & $5.20 \pm 3.25$ & & \\
no & $211(43)$ & $5.45 \pm 3.42$ & & \\
Radiotherapy & & & 0.993 & 0.321 \\
\hline yes & $334(68)$ & $5.41 \pm 3.38$ & & \\
\hline no & $158(32)$ & $5.09 \pm 3.21$ & & \\
\hline
\end{tabular}

\section{Correlation between continuous variables and sleep quality}

Correlation coefficients between continuous variables were presented in Table 2. The mean score of sleep disorders among BC patients was $5.30 \pm 3.32$. The level of sleep disorders was positively correlated with perceived stress $(r=0.168, P<0.01)$, and loneliness $(r=0.158, P<0.01)$. Resilience was negatively associated with sleep disorders $(r=-0.466, P<0.01)$ and negatively correlated with perceived stress $(r=$ $-0.208, P<0.01)$, and loneliness $(r=-0.122, P<0.01)$.

Table 2 Correlations of continuous variables

\begin{tabular}{|lllllll|}
\hline Variables & Mean \pm SD & $\mathbf{1}$ & $\mathbf{2}$ & $\mathbf{3}$ & $\mathbf{4}$ & $\mathbf{5}$ \\
\hline 1. Age (years) & $55.35 \pm 10.57$ & 1 & & & & \\
\hline 2.Sleep disorders & $5.30 \pm 3.32$ & -0.081 & 1 & & & \\
\hline 3.Perceived stress & $19.58 \pm 3.12$ & -0.07 & $0.168^{\star \star}$ & 1 & & \\
\hline 4.Loneliness & $5.00 \pm 1.65$ & $-0.171^{\star \star}$ & $0.158^{\star \star}$ & $0.389^{* \star}$ & 1 & \\
\hline 5.Resilience & $75.76 \pm 11.83$ & $0.104^{\star}$ & $-0.466^{\star \star}$ & $-0.208^{\star \star}$ & $-0.122^{* \star}$ & 1 \\
\hline${ }^{*} P<0.05 ;$ & & & & & & \\
\hline${ }^{* \star} P<0.01$ (two-tailed). & & & & & & \\
\hline
\end{tabular}

\section{Hierarchical multiple linear regression}


The results of hierarchical regression analyses were shown in Table 3 and 4, respectively. Firstly, the control variables (age, principal caregiver) significantly explained sleep disorders (adjusted $R^{2}=0.014$, $\left.\Delta R^{2}=0.020, P<0.05\right)$. In step 2 , perceived stress and loneliness were significantly positive related to sleep disorders, separately $(\beta=0.082, P<0.05 ; \beta=0.119, P<0.01)$, while resilience was significantly and negatively associated with sleep disorders as shown in Table 3 and 4 ( $\beta=-0.452, P<0.01 ; \beta=-0.455, P<$ 0.01). Perceived stress and resilience improved the model fits of sleep disorders (adjusted $R^{2}=0.213, \Delta R^{2}$ $=0.218, P<0.01$ ), loneliness and resilience alleviated the model fits of sleep disorders (adjusted $R^{2}=$ $\left.0.238, \Delta R^{2}=0.225, P<0.01\right)$. The perceived stress $\times$ resilience interaction term was significantly and negatively associated with sleep disorders $(\beta=-0.103, P<0.01)$ in step 3. Simple slope analysis revealed that when resilience is higher, the association between perceived stress and sleep disorders becomes weaker. That is, the impacts of perceived stress on sleep disorders were different in low (1 SD below the mean, $\beta=0.188, P<0.01)$, mean $(\beta=0.110, P<0.01)$ and high (1 SD above the mean, $\beta=0.032, P<0.01)$ levels of resilience. The interaction is visualized in Figure 1 ; the loneliness $\times$ resilience interaction term was also significantly and negatively associated with sleep disorders $(\beta=-0.133, P<0.01)$ in step 3 . Simple slope analysis revealed that when resilience is higher, the association between loneliness and sleep disorders becomes weaker. In other words, the impacts of loneliness on sleep disorders were different in low (1 SD below the mean, $\beta=0.279, P<0.01)$, mean $(\beta=0.135, P<0.01)$ and high (1 SD above the mean, $\beta=-0.01, P<0.01$ ) levels of resilience. The interaction is visualized in Figure 2 .

Table 3 Regression analyses of perceived stress, resilience on sleep disorders 


\begin{tabular}{|c|c|c|c|}
\hline Variables & Step $1(\beta)$ & \multicolumn{2}{|c|}{ Step $2(\beta)$ Step $3(\beta)$} \\
\hline Age (years) & $-0.092^{*}$ & 0.023 & 0.015 \\
\hline \multicolumn{4}{|l|}{ Principal caregiver } \\
\hline caregiver-1 & $-0.124^{*}$ & $-0.123^{*}$ & $-0.117^{\star \star}$ \\
\hline caregiver-2 & -0.020 & $-0.093^{*}$ & $-0.104^{*}$ \\
\hline Perceived stress & & $0.082^{*}$ & $0.109^{\star \star}$ \\
\hline Resilience & & $-0.452^{\star \star}$ & $-0.443^{* *}$ \\
\hline Perceived stress $\times$ Resilience & & & $-0.103^{* *}$ \\
\hline$F$ & $3.376^{*}$ & $30.466^{\star \star}$ & $26.694^{\star \star}$ \\
\hline Adjusted $R^{2}$ & 0.014 & 0.231 & 0.239 \\
\hline$\Delta \mathrm{R}^{2}$ & 0.020 & 0.218 & 0.010 \\
\hline \multicolumn{4}{|c|}{ Note: "Caregiver-1" means "relatives" vs. "spouse", "Caregiver-2" means "adult children” vs. "spouse" } \\
\hline \multicolumn{4}{|l|}{${ }^{*} P<0.05$} \\
\hline${ }^{\star \star} P<0.01$ (two-tailed). & & & \\
\hline
\end{tabular}

Table 4 Regression analyses of loneliness, resilience on sleep disorders 


\begin{tabular}{|c|c|c|c|}
\hline \multirow{2}{*}{$\begin{array}{l}\text { Variables } \\
\text { Age (years) }\end{array}$} & \multirow{2}{*}{$\begin{array}{l}\text { Step } 1(\beta) \\
-0.092^{\star}\end{array}$} & \multicolumn{2}{|c|}{ Step $2(\beta)$ Step $3(\beta)$} \\
\hline & & -0.008 & -0.009 \\
\hline \multicolumn{4}{|l|}{ Principal caregiver } \\
\hline caregiver-1 & $-0.124^{*}$ & $-0.135^{*}$ & $-0.129^{\star \star}$ \\
\hline caregiver-2 & -0.002 & $-0.096^{*}$ & $-0.094^{*}$ \\
\hline Loneliness & & $0.119^{\star *}$ & $0.134^{\star *}$ \\
\hline Resilience & & $-0.455^{\star \star}$ & $-0.443^{\star \star}$ \\
\hline Loneliness $\times$ Resilience & & & $-0.133^{\star \star}$ \\
\hline$F$ & $3.376^{*}$ & $31.647^{* \star}$ & $28.810^{* *}$ \\
\hline Adjusted $R^{2}$ & 0.014 & 0.238 & 0.254 \\
\hline$\Delta \mathrm{R}^{2}$ & 0.020 & 0.225 & 0.017 \\
\hline \multicolumn{4}{|c|}{ Note: "Caregiver-1" means "relatives" vs. "spouse", "Caregiver-2" means "adult children" vs. "spouse" } \\
\hline \multicolumn{4}{|l|}{${ }^{*} P<0.05$} \\
\hline${ }^{* \star} P<0.01$ (two-tailed). & & & \\
\hline
\end{tabular}

\section{Discussion}

This study examined the association between perceived stress, loneliness and sleep disorders respectively, as well as explored whether resilience (a protective factor) could moderate the relationship between perceived stress, loneliness and sleep disorders among Chinese female BC patients. In our study, the prevalence of sleep disorders in female BC patients was $36.58 \%$ and it was much higher than the prevalence of general population in China (14.39\%) [24], higher than the prevalence in Korea $(16.61 \%)$ [22], higher than the prevalence of a previous study among adult female BC patients in Australia (17.5\%) [40] and also higher than the prevalence in America (18.64\%) [8]. There might be several reasons for the situation. First of all, the negative psychological emotions or concomitant symptoms associated with the disease have not been eased. Specifically, psychological stress and discomfort following surgery contributed to sleep disturbances in BC patients [23]. In addition, lacking of professional psychological nursing staff and counseling institutions to disseminate knowledge to patients about how to alleviate cancer-related adverse symptoms like sleep disorders. Consequently, the problem of sleep quality among $\mathrm{BC}$ patients are worthy of concern as well as need to get resolved in time.

The results also revealed that perceived stress and loneliness were significantly correlated with sleep disorders. Women diagnosed with BC would give rise to a series of stressors, including fear of disease 
progression, unexpected side effects and psychologically unpleasant emotional experience [31]. Psychological stress could have a profound impact on sleep, such as causing insomnia or dyssomnia [29]. Indeed, sleep disorders were more commonly when patients with stronger and frequent stress response [28]. Hence, healthcare providers should target for various forms of stress reduction interventions which can relieve sleep disorders for female BC patients. Furthermore, loneliness was also associated with sleep disorders. Cancer-related loneliness is mostly attributed to unmet social involvement or expectations in personal interactions [1]. Loneliness prompted the perception of social threats, which might result in poor health outcomes and stronger sense of stress as well as heighten adverse symptoms such as fatigue, sleep disturbances [2]. Scholars have demonstrated loneliness leads to increasing daytime dysfunction and nightly micro-awakenings, thus indicating the linkage of loneliness with sleep disorders [27]. Overall, interventions related to loneliness should be given more attention to mitigating negative impact on $\mathrm{BC}$ patients.

In this study, resilience was a positive and protective psychology resource for sleep disorders. Additionally, resilience was found to moderate the association of perceived stress and loneliness with sleep disorders. When taking the interaction of perceived stress, loneliness and resilience respectively into the model, it was obvious that the interaction had a significant effect on sleep disorders. Simple slope analysis also revealed that when resilience was higher, the association between perceived stress, loneliness and sleep disorders becomes weaker separately. That is, the results suggested that when women experience a traumatic event and have lower level of resilience, perceived stress aggravated their sleep disorders. Inversely, individuals with higher resilience serves themselves as tenacious and are adaptable to challenging events, which help them with their sleep problems [9]. Similarly, the lonelinesssleep disorders relationship was much stronger for those with lower resilience in comparison to those with high levels of resilience. Hence, interactions indicated that the sensitivity among female breast cancer patients to the effects of perceived stress and loneliness on sleep disorders depends on the positive psychological factor of resilience. The results are in line with previous studies, which found in the COVID-19 context of loneliness-sleep problems [14] and the result of the study on gastrointestinal cancer patients [38], that both perceived stress or loneliness and resilience predict sleep disorders by an interactive manner.

\section{Limitations}

Three limitations of our study should be noted. First, conclusions on the causality of the linkages observed among perceived stress, loneliness, resilience and sleep quality could not be drawn as a result of the cross-sectional design. Second, patients were recruited from a single hospital, Liaoning Province, northeast China, it still needs further studies to be carried out to examine the external generalization of our results among breast cancer patients in different regions or cultural backgrounds. Third, all the variables were measured by using self-report questionnaires, which could be vulnerable to retrospective bias. Further research should consider multiple methodologies to assessing sleep quality.

\section{Clinical implications}

Page 12/19 
Results of our study have implications for future research and clinical nursing. Firstly, clinical nurses should pay attention to the impact that intrinsic psychological symptoms have on sleep quality after diagnosis of $\mathrm{BC}$ in order to alleviate sleep disturbances in this population. For this purpose, consultation by psychologists and mental health professionals should be offered to the patients with poor sleep quality. Secondly, social-psychological factors (perceived stress and loneliness) ought to be considered as one of the most dominating prevention indicator for cancer patients, which may be a key missing component in nursing process $[2,37]$. More concretely, the psychological demand regarding the course of the patients' treatment and care should be combined with to relieve their negative emotion and improve sleep quality. Finally, future research needs to be tailored to some positive psychological variables (e.g., resilience) in the development of improving cancer patients' health interventions.

\section{Conclusions}

In summary, the prevalence of sleep disorders among female BC patients in our study was high. Perceived stress and loneliness were positively associated with sleep disorders, and resilience was negatively associated with sleep disorders, it could relieve the association between perceived stress, loneliness and sleep disorders. In addition to providing resilience training, positive psychological intervention should be integrated into the prevention of sleep quality among Chinese BC patients.

\section{Declarations}

\section{Funding}

This work was supported by National Natural Science Foundation of China [grant number: 71904204].

\section{Acknowledgments}

The authors would like to thank the oncologists in a tertiary cancer hospital in Anshan (Liaoning, China), who helped to get the written informed consent about the conduct of this survey and to distribute the questionnaires to the patients.

\section{Conflict of Interest Statement}

The authors declare that they have no conflicts of interest.

\section{Availability of data and material}

Not applicable

\section{Code availability}

Not applicable.

\section{Authors' contributions}


Yue Ban: Field investigation, data curation and analysis, literature retrieval, writing the article. Mengyao Li: Conceptual design, methodology, formal analysis, revising the paper. He Bai: Data collection. Li Liu, Zhihui Gu, Ke Zhang \& Chenxin Yang: Check and revise the article. Hui Wu: Supervision, project administration, reviewing. All authors reviewed the manuscript and contributed intellectually. The final manuscript was approved by all authors.

\section{Ethic approval}

The protocol for this study was approved by the Ethics Committee of China Medical University (grant number: 71904204). All participants were voluntary in this current research and written informed consent was obtained from each subject.

\section{Consent to participate}

Informed consent was obtained from all individual participants included in the study.

\section{Consent for publication}

We affirm that the participants provided informed consent for publication, as seen in tables $1,2,3,4$ and figure 1 , figure 2 .

\section{References}

1. Adams RN, Mosher CE, Rand KL, Hirsh AT, Monahan PO, Abonour R, Kroenke K (2017) The cancer Ioneliness scale and cancer-related negative social expectations scale: development and validation. Qual Life Res 26: 1901-1913. http://doi.org/10.1007/s11136-017-1518-4

2. Adams RN, Mosher CE, Winger JG, Abonour R, Kroenke K (2018) Cancer-related loneliness mediates the relationships between social constraints and symptoms among cancer patients. J Behav Med 41: 243-252.http://doi.org/10.1007/s10865-017-9892-5

3. Budhrani PH, Lengacher CA, Kip KE, Tofthagen C, Jim H (2014) Minority breast cancer survivors: the association between race/ethnicity, objective sleep disturbances, and physical and psychological symptoms. Nurs Res Pract 2014: 858403.http://doi.org/10.1155/2014/858403

4. Buysse DJ, Reynolds CF, Monk TH, Berman SR, Kupfer DJ (1989) The Pittsburgh sleep quality index: a new instrument for psychiatric practice and research. Psychiatry Research 28: 193213.http://doi.org/10.1016/0165-1781(89)90047-4

5. Carli V, Roy A, Bevilacqua L, Maggi S, Cesaro C, Sarchiapone M (2011) Insomnia and suicidal behaviour in prisoners. Psychiatry Res 185: 141-144. http://doi.org/10.1016/j.psychres.2009.10.001

6. Cohen S, Kamarck T, Mermelstein R (1983) A global measure of perceived stress. Journal of Health and Social Behavior 24:385-96. http://doi.org/10.2307/2136404

7. Deckx L, van den Akker M, Buntinx F (2014) Risk factors for loneliness in patients with cancer: a systematic literature review and meta-analysis. European Journal of Oncology Nursing 18: 466- 
477.http://doi.org/10.1016/j.ejon.2014.05.002

8. Desai K, Mao JJ, Su I, Demichele A, Li Q, Xie SX, Gehrman PR (2013) Prevalence and risk factors for insomnia among breast cancer patients on aromatase inhibitors. Support Care Cancer 21: 4351.http://doi.org/10.1007/s00520-012-1490-z

9. Downing MJ, Houang ST, Scheinmann R, Yoon IS, Chiasson MA, Hirshfield S (2016) Engagement in care, psychological distress, and resilience are associated with sleep quality among HIV-positive gay, bisexual, and other men who have sex with men. Sleep Health 2: 322329.http://doi.org/10.1016/j.sleh.2016.08.002

10. Favez N, Cairo Notari S, Charvoz L, Notari L, Ghisletta P, Panes Ruedin B, Delaloye JF (2016) Distress and body image disturbances in women with breast cancer in the immediate postsurgical period: the influence of attachment insecurity. J Health Psychol 21: 2994-3003. http://doi.org/10.1177/1359105315589802

11. Folkman S, Lazarus RS, Dunkel-Schetter C, DeLongis A, Gruen RJ (1986) Dynamics of a stressful encounter: cognitive appraisal, coping, and encounter outcomes. J Pers Soc Psychol 50: 992-1003. http://doi.org/10.1037//0022-3514.50.5.992

12. Ghoncheh M, Pournamdar Z, Salehiniya H (2016) Incidence and mortality and epidemiology of breast cancer in the world. Asian Pacific Journal of Cancer Prevention 17: 43-46. http://doi.org/10.7314/apjcp.2016.17.S3.43

13. Gray TF, Azizoddin DR, Nersesian PV (2020) Loneliness among cancer caregivers: a narrative review. Palliat Support Care 18: 359-367. http://doi.org/10.1017/S1478951519000804

14. Grossman ES, Hoffman YSG, Palgi Y, Shrira A (2021) COVID-19 related loneliness and sleep problems in older adults: worries and resilience as potential moderators. Pers Individ Dif 168 : 110371.http://doi.org/10.1016/j.paid.2020.110371

15. Gu F, Li XF, Xu JF, Gao GH, Wu YF, Zhou CC (2018) Effect of nicotine dependence on quality of life and sleep quality in patients with lung cancer who continue to smoke after diagnosis. $J$ Thorac Dis 10: 2583-2589. http://doi.org/10.21037/jtd.2018.05.12

16. Hawkley LC, Cacioppo JT (2010) Loneliness matters: a theoretical and empirical review of consequences and mechanisms. Ann Behav Med 40: 218-227.http://doi.org/10.1007/s12160-0109210-8

17. Haynes SN, Adams A, Franzen M (1981) The effects of presleep stress on sleep-onset insomnia. J Abnorm Psychol 90: 601-606. http://doi.org/10.1037//0021-843x.90.6.601

18. Hou WK, Law CC, Yin J, Fu YT (2010) Resource loss, resource gain, and psychological resilience and dysfunction following cancer diagnosis: a growth mixture modeling approach. Health Psychol 29: 484-495. http://doi.org/10.1037/a0020809

19. Hughes ME, Waite LJ, Hawkley LC, Cacioppo JT (2004) A short scale for measuring loneliness in large surveys: results from two population-based studies. Res Aging 26: 655-

672.http://doi.org/10.1177/0164027504268574

Page 15/19 
20. Jaremka LM, Fagundes CP, Glaser R, Bennett JM, Malarkey WB, Kiecolt-Glaser JK (2013) Loneliness predicts pain, depression, and fatigue: understanding the role of immune dysregulation. Psychoneuroendocrinology 38: 1310-1317. http://doi.org/10.1016/j.psyneuen.2012.11.016

21. Kasparian NA (2013) Psychological stress and melanoma: are we meeting our patients' psychological needs?Clin Dermatol 31: 41-46. http://doi.org/10.1016/j.clindermatol.2011.11.005

22. Kim SH, Son BH, Hwang SY, Han W, Yang JH, Lee S, Yun YH (2008) Fatigue and depression in disease-free breast cancer survivors: prevalence, correlates, and association with quality of life. $J$ Pain Symptom Manage 35: 644-655. http://doi.org/10.1016/j.jpainsymman.2007.08.012

23. Kwak A, Jacobs J, Haggett D, Jimenez R, Peppercorn J (2020) Evaluation and management of insomnia in women with breast cancer. Breast Cancer Res Treat 181: 269-

277.http://doi.org/10.1007/s10549-020-05635-0

24. Li N, Xu G, Chen G, Zheng X (2020) Sleep quality among Chinese elderly people: a population-based study. Arch Gerontol Geriatr 87: 103968. http://doi.org/10.1016/j.archger.2019.103968

25. Matthews T, Danese A, Gregory AM, Caspi A, Moffitt TE, Arseneault L (2017) Sleeping with one eye open: Ioneliness and sleep quality in young adults. Psychol Med 47: 2177-

2186.http://doi.org/10.1017/S0033291717000629

26. Montazeri A, Groarke A, Curtis R, Skelton J, Groarke JM (2020) Quality of life and adjustment in men with prostate cancer: Interplay of stress, threat and resilience. Plos One 1510.1371/journal.pone.0239469

27. Mushtaq R (2014) Relationship between loneliness, psychiatric disorders and physical health ? a review on the psychological aspects of Ioneliness. Journal of Clinical and Diagnostic Research $10.7860 / \mathrm{jcdr} / 2014 / 10077.4828$

28. Otte JL, Carpenter JS, Russell KM, Bigatti S, Champion VL (2010) Prevalence, severity, and correlates of sleep-wake disturbances in long-term breast cancer survivors. J Pain Symptom Manage 39: 535547. http://doi.org/10.1016/j.jpainsymman.2009.07.004

29. Palesh O, Aldridge-Gerry A, Ulusakarya A, Ortiz-Tudela E, Capuron L, Innominato PF (2013) Sleep disruption in breast cancer patients and survivors. J Natl Compr Canc Netw 11: 15231530.http://doi.org/10.6004/jnccn.2013.0179

30. Rutter M (1987) Psychosocial resilience and protective mechanisms. Am J Orthopsychiatry 57: 316331.http://doi.org/10.1111/j.1939-0025.1987.tb03541.x

31. Syrowatka A, Motulsky A, Kurteva S, Hanley JA, Dixon WG, Meguerditchian AN, Tamblyn R (2017) Predictors of distress in female breast cancer survivors: a systematic review. Breast Cancer Res Treat 165: 229-245. http://doi.org/10.1007/s10549-017-4290-9

32. Tian J, Hong JS (2013) Validation of the Chinese version of the resilience scale and its cutoff score for detecting low resilience in Chinese cancer patients. Support Care Cancer 21: 14971502.http://doi.org/10.1007/s00520-012-1699-x

33. Tu PC, Yeh DC, Hsieh HC (2020) Positive psychological changes after breast cancer diagnosis and treatment: the role of trait resilience and coping styles. J Psychosoc Oncol 38: 156-170. 
http://doi.org/10.1080/07347332.2019.1649337

34. Wagnild GM (2009) The resilience scale user's guide: for the US English version of the resilience scale and the 14-item resilience scale (RS-14). Montana: Resilience Center 47: 28-33. http://doi.org/10.3928/02793695-20091103-01

35. Wang P, Song L, Wang K, Han X, Cong L, Wang Y, Zhang L, Yan Z, Tang S, Du Y (2019) Prevalence and associated factors of poor sleep quality among Chinese older adults living in a rural area: a population-based study. Aging Clinical and Experimental Research 32: 125-131. http://doi.org/10.1007/s40520-019-01171-0

36. Wang Z, Chen J, Boyd JE, Zhang H, Jia X, Qiu J, Xiao Z (2011) Psychometric properties of the Chinese version of the Perceived Stress Scale in policewomen. PLoS One 6: e28610.http://doi.org/10.1371/journal.pone.0028610

37. Yeung NCY, Ramirez J, Lu Q (2017) Perceived stress as a mediator between social constraints and sleep quality among Chinese American breast cancer survivors. Support Care Cancer 25: 22492257.http://doi.org/10.1007/s00520-017-3632-9

38. Yumei Chen, Hui Yao, Shuying Yan, Yanfen Liu, Lu Y (2019) Effect of emotional resilience group training on fatigue and sleep quality of patients with gastrointestinal cancer. Behavioral Medicine and Brain Science 28: 138-143. http://doi.org/10.3760/cma.j.issn.1674-6554.2019.02.008

39. Zou G, Li Y, Xu R, Li P (2018) Resilience and positive affect contribute to lower cancer-related fatigue among Chinese patients with gastric cancer. J Clin Nurs 27: e1412-e1418. http://doi.org/10.1111/jocn.14245

40. Zucca AC, Boyes AW, Linden W, Girgis A (2012) All's well that ends well? Quality of life and physical symptom clusters in long-term cancer survivors across cancer types. J Pain Symptom Manage 43: 720-731. http://doi.org/10.1016/j.jpainsymman.2011.04.023

\section{Figures}




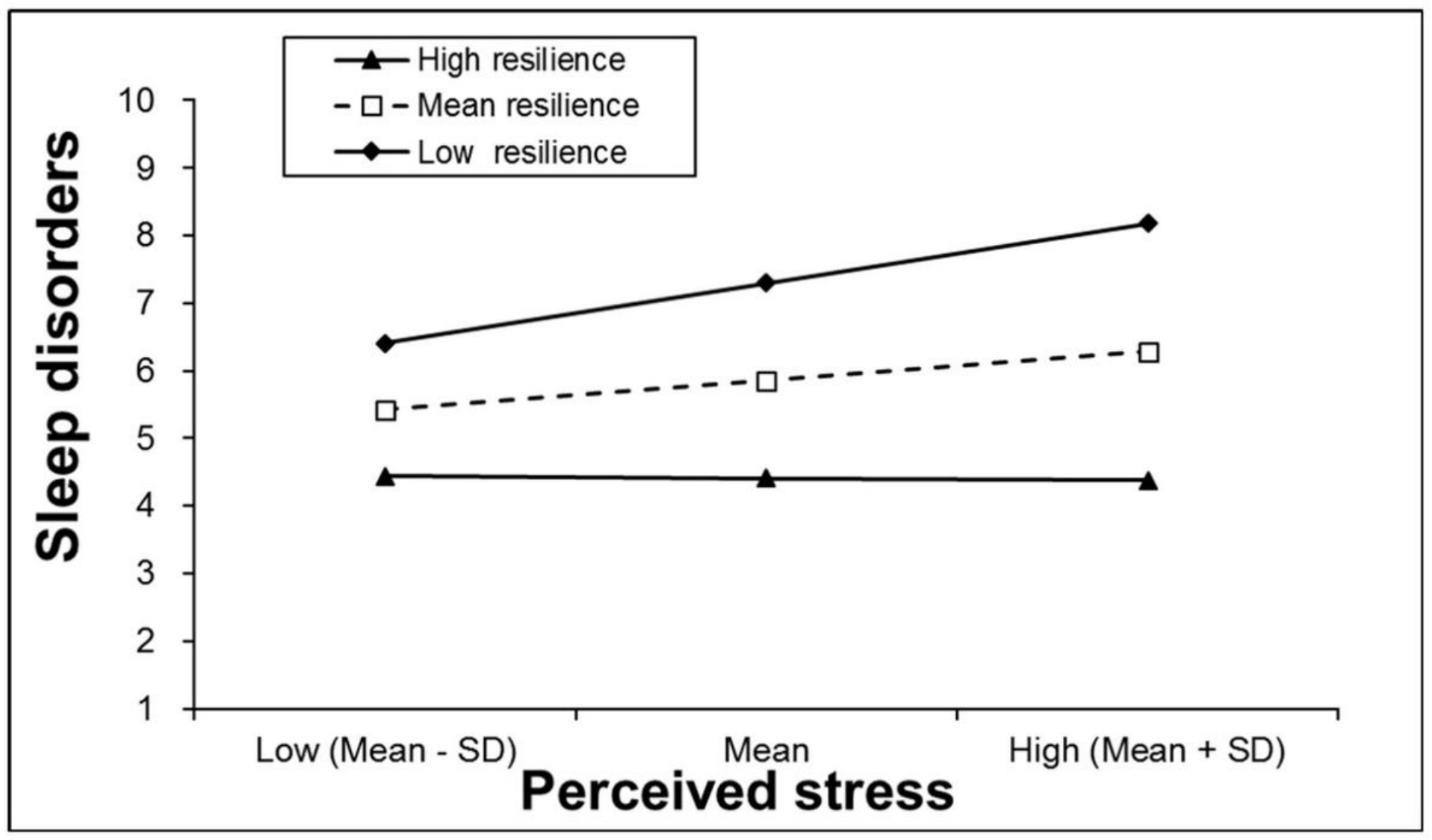

Figure 1

Simple slope plot of interaction between perceived stress and resilience on sleep disorders $(N=492)$. Note: Low, 1 SD below the mean; high, 1 SD above the mean. Abbreviation: SD, standard deviation. 


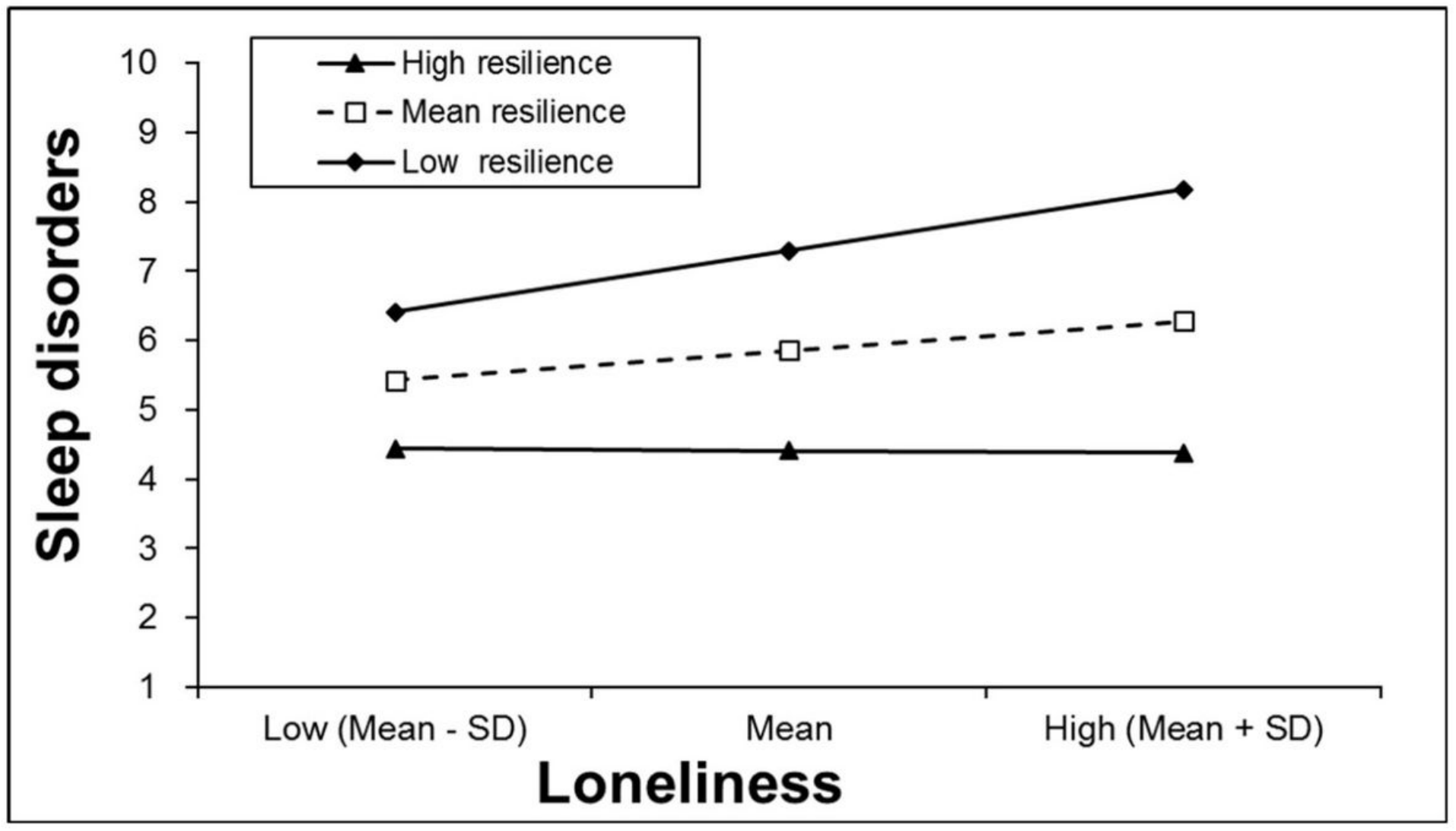

Figure 2

Simple slope plot of interaction between loneliness and resilience on sleep disorders $(N=492)$. Note: Low, 1 SD below the mean; high, 1 SD above the mean. Abbreviation: SD, standard deviation. 